\title{
Renoprotective mechanisms of pirfenidone in hypertension-induced renal in- jury: through anti-fibrotic and anti-oxidative stress pathways
}

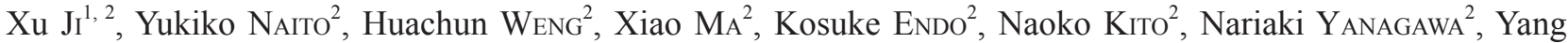 \\ $\mathrm{Yu}^{1}$, Jie $\mathrm{LI}^{1}$, and Naoharu IwAI ${ }^{2}$ \\ ${ }^{1}$ State Key Laboratory of Phytochemistry and Plant Resources in West China, Kunming Institute of Botany, Chinese Academy of Sci- \\ ences, Kunming, Yunnan 650201, China and ${ }^{2}$ Department of Genomic Medicine, National Cerebral and Cardiovascular Center, Suita \\ 5658565 , Japan
}

(Received 30 September 2013; and accepted 17 October 2013)

\begin{abstract}
Pirfenidone (PFD) is a novel anti-fibrotic agent that targets TGF $\beta$. However, the mechanisms underlying its renoprotective properties in hypertension-induced renal injury are poorly understood. We investigated the renoprotective properties of PFD and clarified its renoprotective mechanisms in a rat hypertension-induced renal injury model. Dahl salt-sensitive rats were fed a high-salt diet with or without $1 \%$ PFD for 6 weeks. During the administration period, we examined the effects of PFD on blood pressure and renal function. After the administration, the protein levels of renal TGF $\beta$, Smad2/3, TNF $\alpha$, MMP9, TIMP1, and catalase were examined. In addition, total serum antioxidant activity was measured. Compared to untreated rats, PFD treatment significantly attenuated blood pressure and proteinuria. Histological study showed that PFD treatment improved renal fibrosis. PFD may exert its anti-fibrotic effects via the downregulation of TGF $\beta$-Smad $2 / 3$ signaling, improvement of MMP9/TIMP1 balance, and suppression of fibroblast proliferation. PFD treatment also increased catalase expression and total serum antioxidant activity. In contrast, PFD treatment did not affect the expression of TNF $\alpha$ protein, macrophage or T-cell infiltration, or plasma interleukin $1 \beta$ levels. PFD prevents renal injury via its anti-fibrotic and anti-oxidative stress mechanisms. Clarifying the renoprotective mechanisms of PFD will help improve treatment for chronic renal diseases.
\end{abstract}

Pirfenidone (PFD; 5-methyl-1-phenylpyridin-2-one) is an anti-fibrotic agent. PFD has been considered as an antagonist of transforming growth factor $\beta$ (TGF $\beta$ ), and has anti-fibrotic, anti-inflammatory, and antioxidative stress activities $(6,12-14,27,36)$. PFD has demonstrated an anti-fibrotic effect via the downregulation of TGF $\beta$ expression in several animal nephropathy models, including unilateral ureteral

Address correspondence to: $\mathrm{Xu} \mathrm{Ji}, \mathrm{PhD}$ or Yukiko Naito, $\mathrm{PhD}$

Department of Genomic Medicine, National Cerebral and Cardiovascular Center, 5-7-1 Fujishirodai, Suita, Osaka 565-8565, Japan

Tel: +81-6-6833-5012 (Ext 2513), Fax: +81-6-6835-2088

E-mail: jixu@mail.kib.ac.cn or naitoy@ncvc.go.jp obstruction (36), diabetic nephropathy $(24,27)$, nephrectomy $(35,38)$, vanadate-induced renal fibrosis (1), and calcineurin inhibitor-induced nephrotoxicity $(5,6,34)$. In addition, the clinical effectiveness of PFD has been evaluated in human diabetic nephropathy (32) and focal segmental glomerulosclerosis (7). The anti-fibrotic effect of PFD might also be due to the regulation of the balance of matrix metalloproteinases (MMPs) and their inhibitors, tissue inhibitors of metalloproteinases (TIMPs) $(6,10,20,36)$. Furthermore, PFD may also exert its anti-fibrotic effect via the suppression of fibroblast proliferation (14).

PFD has demonstrated anti-inflammatory effects via the inhibition of the expressions of inflammatory mediators such as tumor necrosis factor $\alpha$ (TNF $\alpha)$ and interleukin $1 \beta$ (IL1 $\beta$ ) both in vivo and vitro (2, 
$12,13,20)$. Oxidative stress is related to progressive renal injury (28). PFD is reported to exert antioxidative stress in mesangial cells and in a cirrhosis model $(27,30)$. Therefore, the anti-inflammatory and anti-oxidative stress effects of PFD are also expected to ameliorate renal injury. At present, although some renoprotective mechanisms of PFD have been reported, no systematic study has clarified the renoprotective mechanisms of PFD. Furthermore, the mechanisms underlying the renoprotective action of PFD in hypertension-induced renal injury are poorly understood. Therefore, the present systematic study investigated the anti-fibrotic, anti-inflammatory, and anti-oxidative stress effects of PFD. To elucidate the mechanisms underlying the renoprotective action of PFD, we administered PFD to Dahl salt-sensitive rats, and measured blood pressure and renal function. We also investigated the effects of PFD on TGF $\beta$ protein levels as well as its upstream and downstream signaling pathways. The PFDinduced protein expression changes of MMP9 and TIMP1 were examined. The effects of PFD on fibroblast proliferation were also investigated by immunohistochemistry. In addition, the effects of PFD on inflammatory factors such as macrophage and Tcell infiltration, TNF $\alpha$ protein expression, and plasma levels of IL1 $\beta$ were examined. Finally, we investigated catalase protein expression and total serum antioxidant activity.

\section{MATERIALS AND METHODS}

Experimental animals. Dahl salt-sensitive rats were obtained from SLC Japan (Shizuoka, Japan). The rats were housed in a temperature-controlled pathogenfree room with light from 0700 to $1900 \mathrm{~h}$ (daytime) and had free access to food and water. Experiments were performed in accordance with the guidelines of the National Institutes of Health Guide for the Care and Use of Laboratory Animals, and the experimental protocols were approved by the National Cerebral and Cardiovascular Center for the Care and Use of Experimental Animals.

Renoprotective effects of PFD in Dahl salt-sensitive rats. Dahl salt-sensitive rats were fed a high-salt diet [8\% $\mathrm{NaCl}(\mathrm{w} / \mathrm{w})$; Oriental Yeast, Tokyo, Japan] from 4.5 weeks of age. PFD-treated Dahl salt-sensitive rats (PFD group, $\mathrm{n}=10$ ) were fed the high-salt diet with mixed with 1\% PFD (approximately $700 \mathrm{mg}$ $\mathrm{kg}^{-1}$ day $^{-1}$ ). Control Dahl salt-sensitive rats (Control group, $\mathrm{n}=10$ ) were administered only the high-salt diet. Rats were treated with PFD for 6 weeks. PFD was provided by Shionogi (Osaka, Japan).

Blood pressure was measured once every 2 weeks using a tail-cuff method (BP-98A; Softron, Tokyo, Japan). Rats were housed in metabolic cages once every 2 weeks for $24-\mathrm{h}$ urine collection. Urinary protein levels were measured using a BCA protein assay kit (Thermo Scientific, Rockford, IL, USA). Creatinine clearance $\left(\mathrm{C}_{\mathrm{Cr}}\right)$ at week 6 of administration was calculated using the following formula: $\mathrm{C}_{\mathrm{Cr}}=\left(\mathrm{U}_{\mathrm{Cr}} \times \mathrm{V}\right) / \mathrm{P}_{\mathrm{Cr}} ; \mathrm{U}_{\mathrm{Cr}}$ is the concentration of urinary creatinine $\left(\mathrm{mg} \cdot \mathrm{mL}^{-1}\right), \mathrm{P}_{\mathrm{Cr}}$ is the concentration of plasma creatinine $\left(\mathrm{mg} \cdot \mathrm{mL}^{-1}\right)$, and $\mathrm{V}$ is the urine flow rate $\left(\mathrm{mL} \cdot \mathrm{min}^{-1}\right)$. Creatinine levels were measured using a QuantiChrom creatinine assay kit (DIUR-500; BioAssay Systems, Hayward, CA, USA).

Measurement of TGF $\beta$ mRNA in the Dahl salt-sensitive rat kidneys. After 6 weeks with or without PFD treatment, the rats were anesthetized with pentobarbital $\left(25 \mathrm{mg} \cdot \mathrm{kg}^{-1}\right)$. Venous blood was collected from the vena cava. Plasma (EDTA as an anticoagulant) and serum were isolated by centrifugation and stored at $-80^{\circ} \mathrm{C}$ until measurement. The left kidneys of the rats were removed, weighed, and sectioned longitudinally. Half of each left kidney was frozen in liquid nitrogen and stored at $-80^{\circ} \mathrm{C}$ until measurement, while the other half was used to isolate RNA with TRIzol reagent (Invitrogen, Carlsbad, CA, USA) according to the manufacturer's instructions. TGF $\beta$ mRNA expression levels were determined by real-time RT-PCR using a commercial kit (Rn01442102_m1; Applied Biosystems, Foster City, CA, USA) and normalized to the expression of $\beta$-actin mRNA; these measurements were expressed as $\log _{10}\left(2^{35-\mathrm{CT} 1} / 2^{25-\mathrm{CT} 2}\right)$, where $2^{35 \text {-CT1 }}$ and $2^{25 \text {-CT2 }}$ correspond to the expression levels of TGF $\beta$ and $\beta$-actin mRNA, respectively, as described previously (17).

Western blotting. Western blot analysis was performed using the extracts from rat kidneys frozen in liquid nitrogen and homogenized in 1\% NP-40 lysis buffer as described previously (15). Equal amounts of protein $(40 \mu \mathrm{g})$ were separated by SDS-PAGE $(10 \%$ gels) and then transferred to a nitrocellulose membrane (Hybond ECL, $0.22 \mu \mathrm{m}$; GE Healthcare, Buckinghamshire, UK) or a PVDF membrane (Hybond-P, $0.45 \mu \mathrm{m}$; GE Healthcare). After blocking with $5 \%(\mathrm{w} / \mathrm{v})$ skim milk powder, the membranes were incubated overnight at $4{ }^{\circ} \mathrm{C}$ with primary antibodies (Santa Cruz Biotechnology, Santa Cruz, CA, USA) against TGF $\beta, \operatorname{Smad} 2 / 3$, phosphorylated $\operatorname{Smad} 2 / 3(\mathrm{pSmad} 2 / 3)$, chromosome $3 \mathrm{p}$ kinase $(3 \mathrm{pK})$, homeodomain-interacting protein kinase 2 (HIPK2), 
TNF $\alpha$, MMP9, TIMP1, and catalase at $1: 500$ dilution. Membrane-bound antibodies were visualized with a horseradish peroxidase-conjugated secondary antibody (Amersham Biosciences, Buckinghamshire, UK) at $1: 10000$ dilution and an ECL advanced Western blotting detection kit (GE Healthcare). $\beta$-Actin (Santa Cruz Biotechnology) was used as a loading control.

Histological examination. As previously reported (16), for histological evaluation, the rats on week 6 of high-salt intake with or without PFD treatment were anesthetized with pentobarbital $\left(25 \mathrm{mg} \cdot \mathrm{kg}^{-1}\right)$ and portions of the right kidney were fixed in $10 \%$ buffered formalin-saline overnight at $4{ }^{\circ} \mathrm{C}$ and subsequently embedded in paraffin blocks. Tissue sections $5-\mu \mathrm{m}$ thick were stained with Masson's trichrome. The area of renal fibrosis was measured and analyzed with a BZ Image Analyzer II (Keyence, Osaka, Japan).

Immunohistochemistry. Immunohistochemistry was used to detect macrophages (CD68), T cells (CD3), and fibroblasts (FSP1, fibroblast-specific protein 1). After deparaffinization and antigen retrieval, endogenous peroxidase activity was blocked by $3.3 \% \mathrm{H}_{2} \mathrm{O}_{2}$ and nonspecific binding was blocked by $5 \%$ normal goat serum. The sections were incubated overnight at $4{ }^{\circ} \mathrm{C}$ with primary antibodies against CD68 (Abcam, Cambridge, UK), CD3 (BD Pharmingen, San Diego, CA, USA), and FSP1 (Abcam). The Simplestain MAX-PO (rat) kit (Nichirei, Tokyo, Japan) was used as a secondary antibody and allowed to incubate for $30 \mathrm{~min}$ at room temperature. Antibody binding was visualized using 3,3'-diaminobenzidine (DAB; Dojindo Laboratories, Kumamoto, Japan), and the nuclei were stained with hematoxylin. The numbers of positive cells were counted, and the results are expressed as the number of positive cells per square millimeter renal tissue.

Plasma IL1 $\beta$ measurement. Plasma IL1 $\beta$ was measured in duplicate with an enzyme-linked immunosorbent assay (ELISA) kit (IBL, Gunma, Japan).

Measurement of total serum antioxidant activity. To evaluate reactive oxygen species, we determined the total serum antioxidant activity by using the total antioxidant power colorimetric microplate assay kit (Oxford Biomedical Research, Oxford, MI, USA), which measures the reductive capacity by detecting the reduction of $\mathrm{Cu}^{2+}$ to $\mathrm{Cu}^{+}$.
Statistical analysis. Values are expressed as mean \pm standard error of the mean (SEM). Statistical analyses were performed using JMP (SAS Institute, Cary, NC, USA). Analysis of variance (ANOVA) was used to estimate differences among groups, and the significance of differences was tested using Student's $t$-test.

\section{RESULTS}

Effects of PFD treatment on body weight, urinary protein, $C_{C r}$, heart rate, and blood pressure

Between the Control and PFD groups, there were no significant differences in body weight (Fig. 1A) and food intake (data not shown). Neither urine volume (Fig. 1B) nor water intake (data not shown) was significantly different between the Control and PFD groups. In contrast, urinary protein excretion was significantly attenuated at weeks 2 and 4 after the PFD treatment, indicating that PFD exerted a renoprotective effect (Fig. 1C). Although a trend demonstrating the improvement of $\mathrm{C}_{\mathrm{Cr}}$ was observed after 6 weeks of PFD treatment (Fig. 1D), there was no significant difference between the Control and PFD groups $\left(1.58 \pm 0.22\right.$ vs. $2.93 \pm 0.66 \mathrm{~mL} \cdot \mathrm{min}^{-1}$, respectively; $P=0.13$ ).

PFD treatment did not affect heart rate as measured by the tail-cuff method (Fig. 1E). Six weeks of $8 \% \mathrm{NaCl}$ diet feeding increased systolic blood pressure (SBP) in the Control group significantly. PFD treatment significantly attenuated this increase in SBP at weeks 2 and 4 (Fig. 1F).

From the results described above, we conclude that PFD treatment ameliorates hypertension and renal injury.

Effects of PFD treatment on renal weight and fibrosis There were no significant differences in renal weight between the Control and PFD groups at week 6 (Fig. 2A). Six weeks of $8 \% \mathrm{NaCl}$ diet feeding caused tubular dilatation, interstitial fibrosis, and glomerular sclerosis (Fig. 2C); PFD treatment significantly attenuated these pathological changes (Fig. 2D). PFD treatment markedly reduced fibrotic areas (Fig. 2B).

Effects of PFD treatment on renal TGF $\beta$, Smad2/3, pSmad2/3, 3pK, and HIPK2 expression

PFD exerts a renoprotective effect via the downregulation of TGF $\beta$ expression $(1,5-7,24,27,34-36$, 38). Therefore, we examined whether PFD regulates TGF $\beta$ expression in a rat salt-sensitive hypertension model. Indeed, PFD treatment significantly reduced the mRNA (Fig. 3A) and protein (Fig. 3B) expres- 
A:

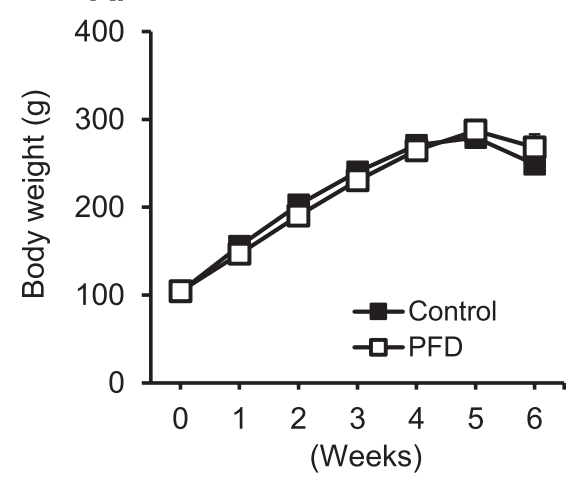

C:

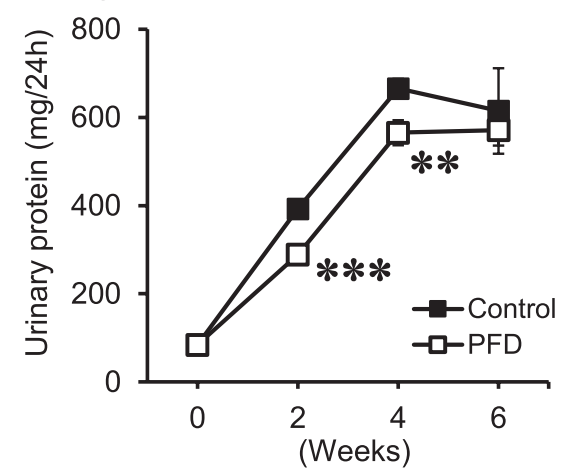

E:

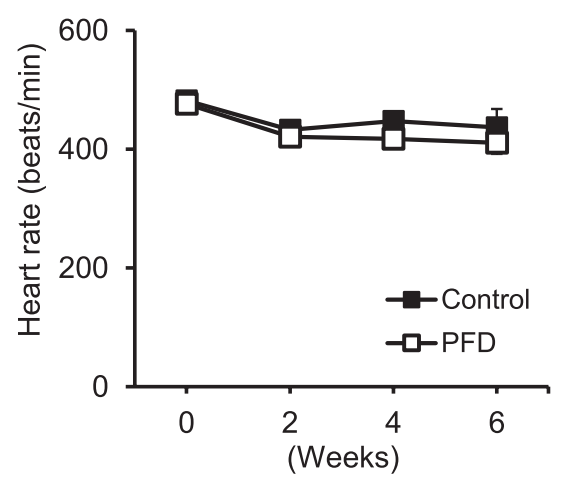

B:

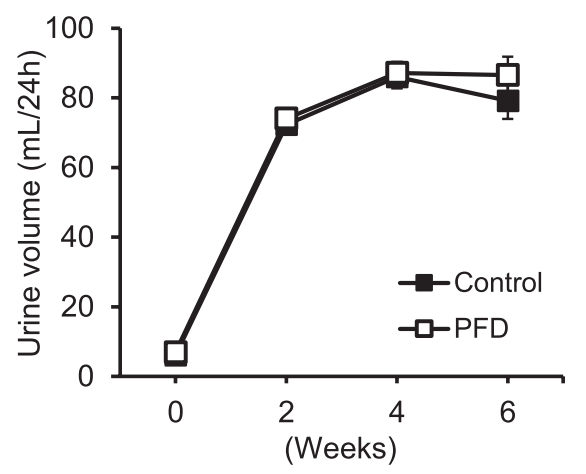

D:

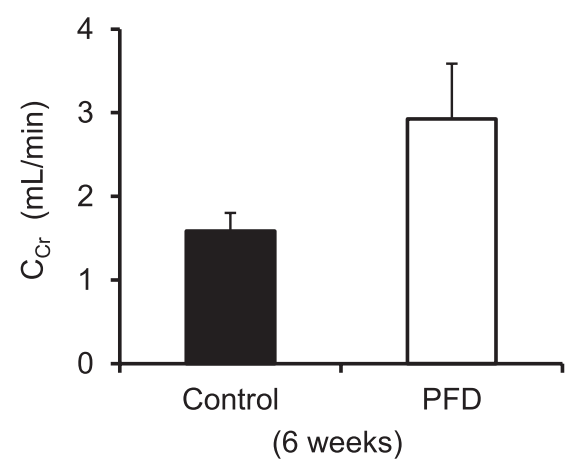

F:

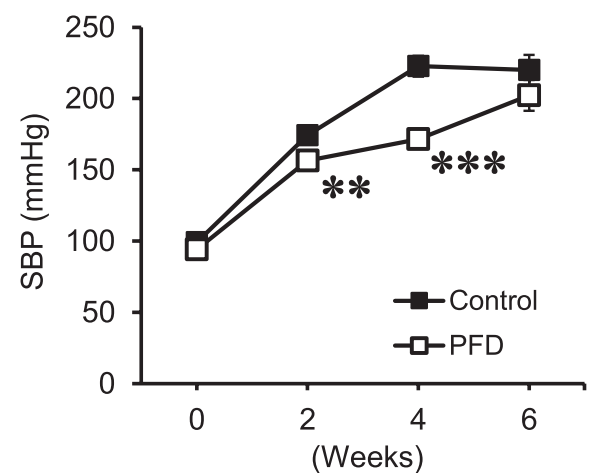

Fig. 1 Effects of PFD treatment on body weight, urinary protein, $\mathrm{C}_{\mathrm{Cr}}$, heart rate, and blood pressure. PFD-treated Dahl salt-sensitive rats (PFD group, $n=10$ ) were fed a high-salt diet mixed with $1 \%$ PFD for 6 weeks. Control Dahl salt-sensitive rats (Control group, $n=10$ ) were administered only the high-salt diet. Heart rate and SBP were measured by the tail-cuff method. There were no significant differences with respect to body weight $(A)$, urine volume $(B), C_{C r}(D)$, or heart rate $(E)$ between the Control and PFD groups. Six weeks of the $8 \% \mathrm{NaCl}$ diet significantly increased urinary protein excretion (C) and SBP (F) in the Control group; PFD treatment significantly attenuated these increases in urinary protein excretion (C) and SBP $(\mathrm{F})$ at weeks 2 and $4 .{ }^{* *} P<0.01,{ }^{* * *} P<0.001$, significantly different from the Control group (unpaired Student's $t$-test).

sions of renal TGF $\beta$ compared to the Control group. TGF $\beta$ activates a unique signal transduction pathway that acts via the Smad family of proteins (9, 27). In the present study, PFD treatment did not affect the protein expression of total Smad2/3 (Fig. 3C). However, PFD treatment significantly attenuated $\mathrm{pSmad} 2 / 3$ protein expression in the kidneys
(Fig. 3D). PFD treatment also attenuated the ratio of $\mathrm{pSmad} 2 / 3$ and total Smad2/3 (Control vs. PFD treatment, $1.40 \pm 0.16$ vs. $0.83 \pm 0.11$, respectively; $P=$ $0.002)$. These results confirm that PFD treatment does not affect total $S m a d 2 / 3$ production but decreases $\operatorname{Smad} 2 / 3$ activity.

TGF $\beta$ also activates Smad-independent signaling 

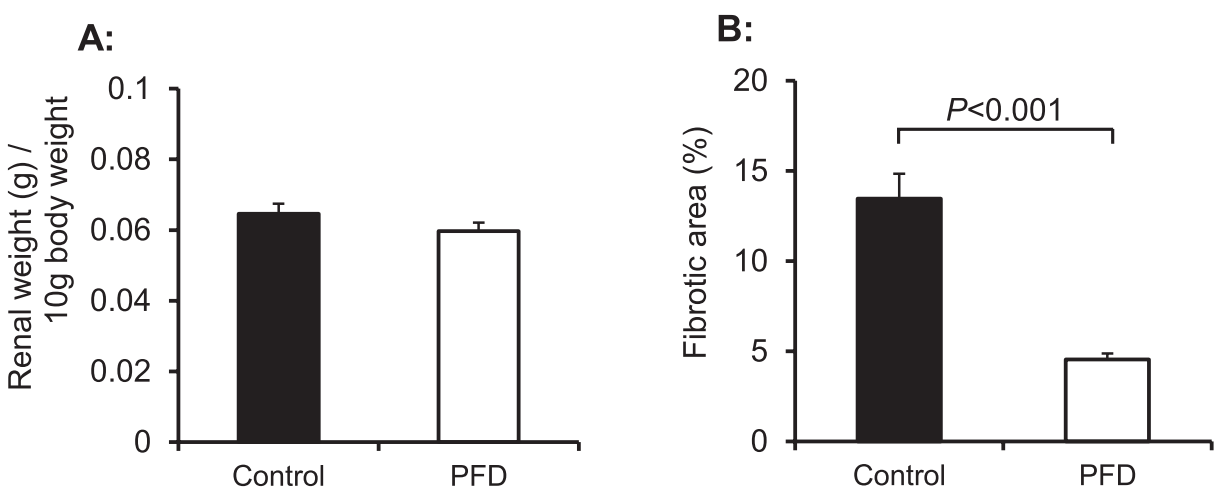

C:

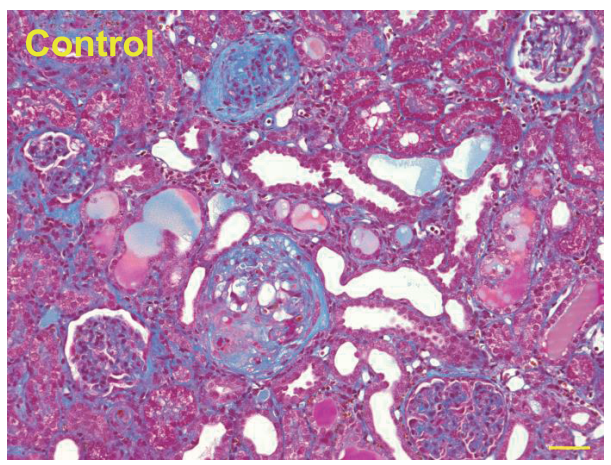

D:

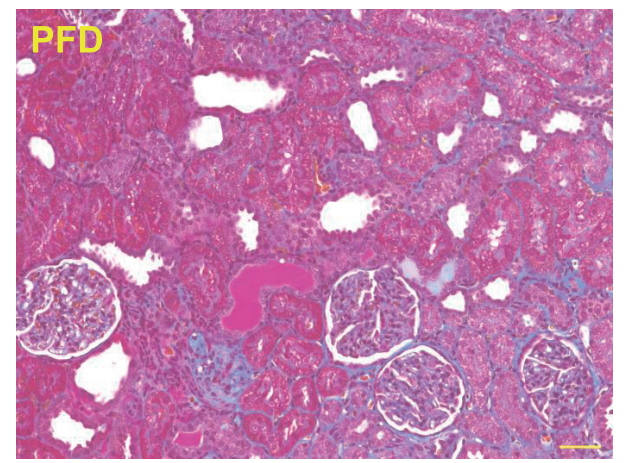

Fig. 2 Effects of PFD treatment on renal weight and fibrosis. (A) Relative renal weight in the Control $(n=10)$ and PFD $(n=10)$ groups at week 6; values represent renal organ weight per $10 \mathrm{~g}$ body weight. Renal weight was not significantly different between the Control and PFD groups. Representative images of the kidneys of the Control (C) and PFD (D) groups stained with Masson's trichrome exhibiting interstitial fibrosis and glomerular sclerosis (blue-colored area). PFD treatment markedly reduced the percentage of fibrotic areas (B).

pathways, including MAPK-family proteins (9). Also known as MAPKAPK3, $3 \mathrm{pK}$ is activated by three members of the MAPK family: extracellular signal-regulated kinase (ERK), p38MAPK, and Jun N-terminal kinase (JNK) (22). In the present study, renal $3 \mathrm{pK}$ protein levels were not significantly different between the Control and PFD groups (Fig. 3E). HIPK2 regulates renal fibrosis by activating the TGF $\beta$ pathway (18). In the present study, there was no significant difference between the Control and PFD groups with respect to HIPK2 protein expression (Fig. 3F).

From the results described above, we conclude that PFD exerts its renoprotective effect via downregulation of the TGF $\beta$ and Smad2/3 signaling pathways.

Effects of PFD treatment on renal MMP/TIMP balance

MMP9 and its main inhibitor, TIMP1, play important roles in renal fibrosis $(11,21,23)$. Nephritis-induced renal injury is more severe in MMP9-deficient mice than wild-type mice (21). Moreover, the significant rise of TIMP1 expression was attenuated by PFD in calcineurin inhibitor-induced nephrotoxicity (6). Therefore, the aim of the present study was to evaluate the effects of PFD treatment on renal MMP9/ TIMP1 balance in hypertensive renal injury. Compared to the Control group, PFD treatment significantly increased renal MMP9 protein expression (Fig. 4A), whereas it significantly decreased that of renal TIMP1 (Fig. 4B).

\section{Effects of PFD treatment on renal proliferation}

FSP1, also called S100A4, is a specific fibroblast marker (37). In the present study, we did not observe significant suppression of fibroblast proliferation in renal interstitium after PFD treatment; we observed PFD treatment significantly attenuated the number of FSP1-positive cells in the fibrosis areas (Fig. 5). 
A:

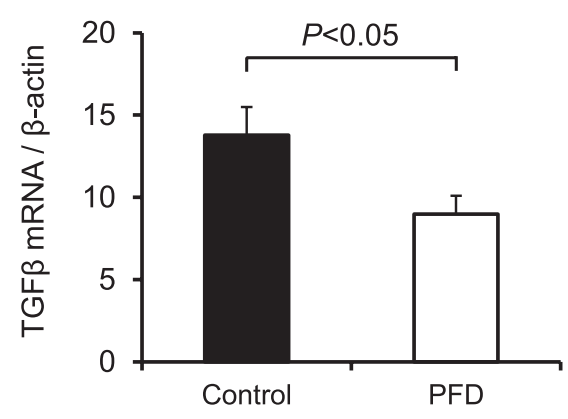

C:

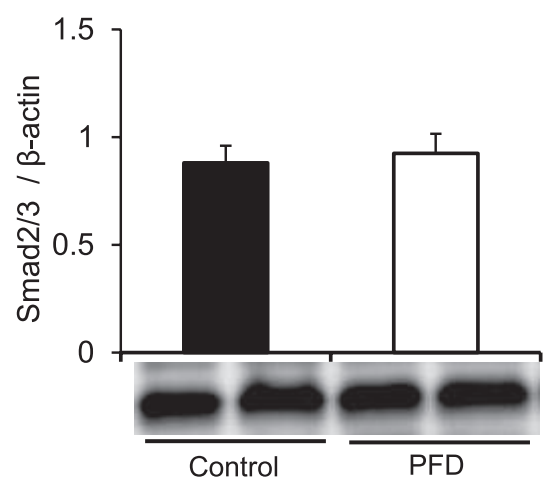

E:

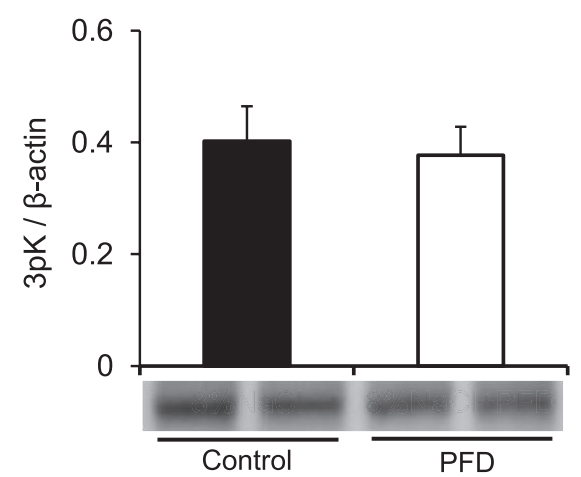

B:

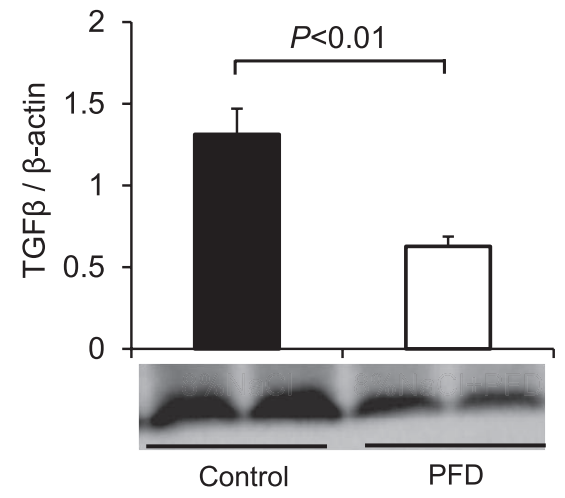

D:

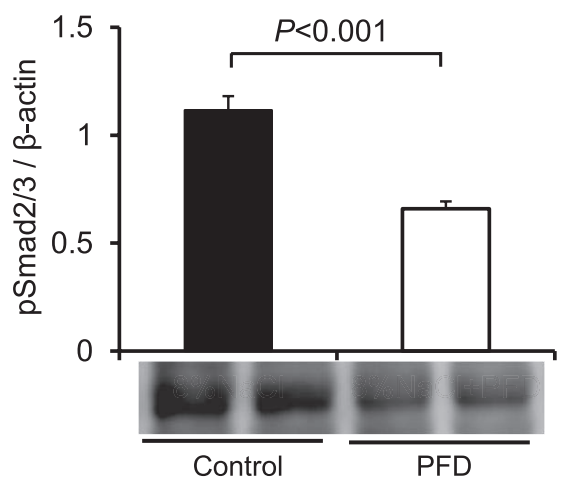

F:

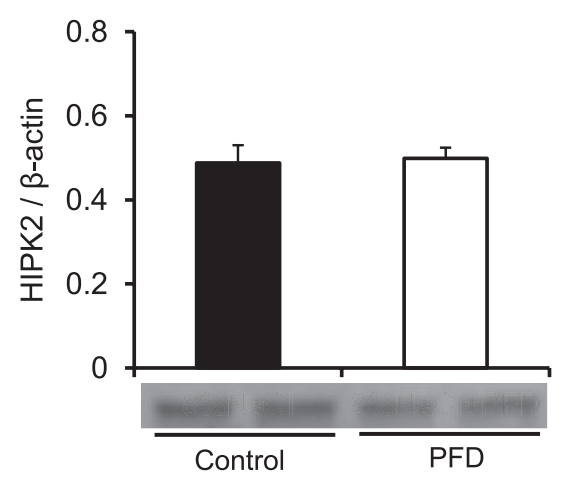

Fig. 3 Effects of PFD treatment on renal TGF $\beta$, Smad2/3, pSmad2/3, 3pK, and HIPK2 expression. Expressions of TGF $\beta$ mRNA (A), TGF $\beta$ protein (B), Smad2/3 protein (C), pSmad2/3 protein (D), 3pK protein (E), and HIPK2 protein (F) in the kidneys of the Control and PFD groups. ( $n=10$ for Control and PFD groups)

Effects of PFD treatment on renal inflammatory reaction

To evaluate the renal inflammation caused by saltinduced hypertension, tissue sections of the kidneys were stained with CD68 (a macrophage marker) and CD3 (a T-cell marker). There were no significant differences between the Control and PFD groups with respect to macrophage (Fig. 6C) or T-cell infiltration (Fig. 6F).

PFD is reported to inhibit TNF $\alpha$ and IL1 $\beta$ expres- sion $(2,12,13,20)$. However, we did not observe significant attenuation of renal TNF $\alpha$ protein expression (Fig. 6G) or plasma IL1 $\beta$ levels (Fig. 6H) after PFD treatment using Western blotting and ELISA, respectively.

\section{Effects of PFD treatment on antioxidant activity}

PFD was reported to have exhibited anti-oxidative stress effects in mesangial cells and in a cirrhosis model $(27,30)$. In the present study, a total antioxi- 

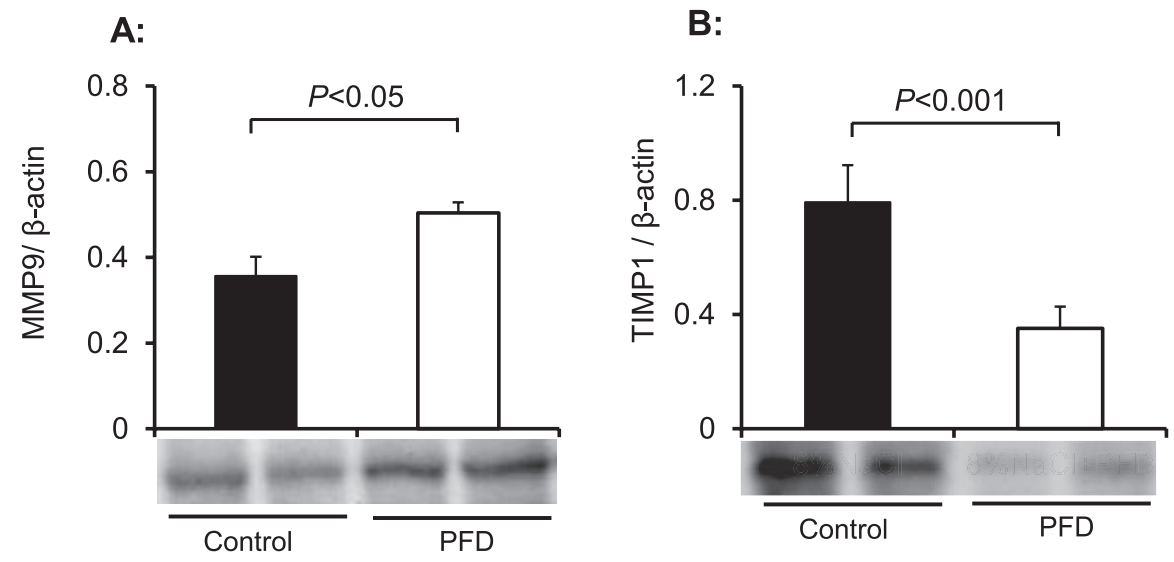

Fig. 4 Effects of PFD treatment on renal MMP/TIMP balance. PFD treatment significantly increased renal MMP9 protein expression (A). Renal TIMP1 protein levels in the PFD group were significantly lower than that in the Control group (B). ( $n=10$ for Control and PFD groups)

dant power kit was used to measure total serum antioxidant activity; we found that PFD treatment significantly increased the total antioxidant activity in serum (Fig. 7A; Control vs. PFD treatment, 10.17 \pm 0.42 vs. $\left.12.88 \pm 0.76 \mathrm{nmol} \cdot \mathrm{mg} \operatorname{protein}^{-1} ; P<0.05\right)$. In addition, Western blotting revealed that PFD treatment significantly increased catalase protein expression in the kidneys (Fig. 7B).

\section{DISCUSSION}

PFD is a novel anti-fibrotic agent that inhibits the progression of renal fibrosis $(1,5-7,24,27,34-36$, 38). PFD has demonstrated anti-fibrotic, anti-inflammatory, and anti-oxidative stress effects in animal models and patients with renal diseases $(6,12-14$, $27,36)$. In the present study, we observed that PFD treatment decreased SBP, reduced proteinuria, and ameliorated renal fibrosis in a rat model of hypertensive renal injury.

TGF $\beta$ is a key protein in renal fibrosis $(3,4,33)$, and PFD is an antagonist of TGF $\beta$. PFD also decreases TGF $\beta$ expression in several animal nephropathy models $(1,5-7,24,27,34-36,38)$. In the present study, we also observed that PFD treatment decreased renal TGF $\beta$ mRNA and protein levels. TGF $\beta$ frequently modulates the transcription of key target genes via the Smad signaling pathway, which directly transduces TGF $\beta$ receptor activation to the nucleus $(4,9,26,31)$. In addition, Smad-independent signaling pathways via ERK, p38MAPK, and JNK provide alternative gene-activation mechanisms $(9,20)$.

In the present study, we observed that PFD treatment decreased Smad2/3 activity. In addition, renal
$3 \mathrm{pK}$ protein levels were not significantly different between the Control and PFD groups. The uniqueness of $3 \mathrm{pK}$ is due to it being a novel convergence point of three MAPK pathways: ERK, p38MAPK, and JNK (22). From the results described above, we conclude that 1) PFD treatment decreases renal TGF $\beta$ expression, which may mediate the downregulation of Smad2/3 activity; and 2) the Smad-independent signaling pathways of ERK, p38MAPK, and JNK are not affected by PFD treatment upon renal injury and fibrosis. Recent reports also indicate that TGF $\beta$ and its downstream Smad pathway play important roles in renal diseases $(4,26,31)$. In particular, PFD inhibits total Smad2/3 protein expression and TGF $\beta$-induced Smad 2 phosphorylation in murine mesangial cells (27). The balance between MMPs and TIMPs is also influenced by TGF $\beta$ in the kidneys $(8,10,25)$. Furthermore, TGF $\beta$ stimulates fibroblast proliferation in the renal interstitium via a Smad-independent signaling pathway (39). From the results of the present and previous studies, we conclude that TGF $\beta$ is a potential central regulator of renal fibrosis. HIPK2 is reported to be a key regulator of renal fibrosis via the activation of the TGF $\beta$-Smad3 pathway (18). However, PFD treatment did not affect HIPK2 protein expression in the kidneys in this study. This result suggests PFD treatment does not affect the TGF $\beta$ upstream signaling of HIPK2.

TIMP1 is the natural inhibitor of MMP9; both of these proteins play pivotal roles in kidney diseases. Therefore, disturbances in the balance of two systems lead to renal fibrosis $(11,21,23)$. Nephritis-induced renal injury is more severe in MMP9-deficient mice than wild-type mice, which indicates that MMP9 
A:

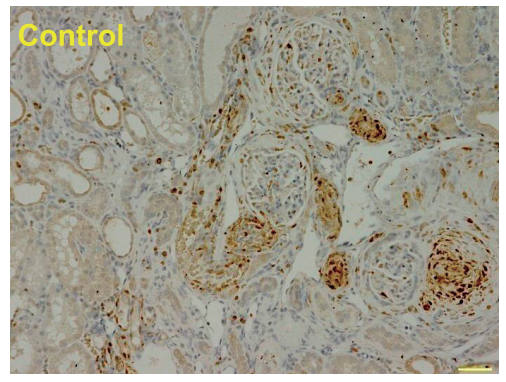

C:

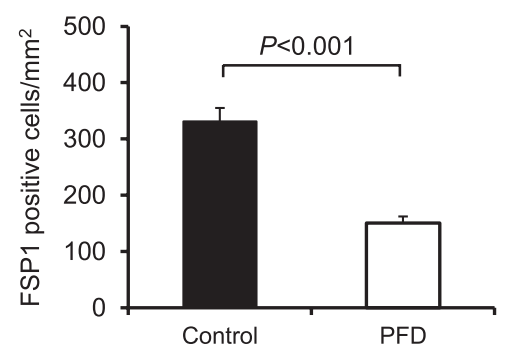

B:

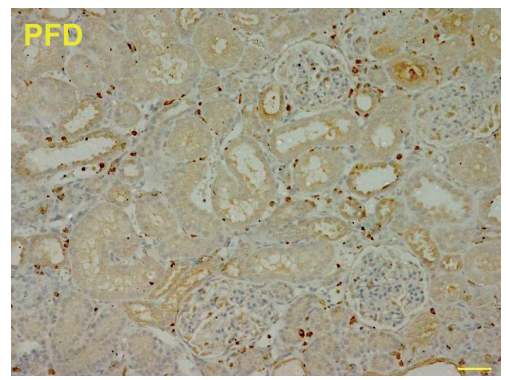

Fig. 5 Effects of PFD treatment on renal fibroblast proliferation. Immunohistochemical examination revealed fibroblasts, which were identified by positive staining for anti-FSP1 (a specific marker of fibroblasts) antibody. FSP1-positive cells were counted and compared between the Control and PFD groups. There were significantly more fibroblasts in the kidneys of the Control group (A) than the PFD group (B). Average numbers of immune cells per square millimeter in the Control $(n=10)$ and PFD $(n=10)$ groups $(C)$. Fibroblast proliferation was significantly attenuated in the PFD group compared to the Control group.

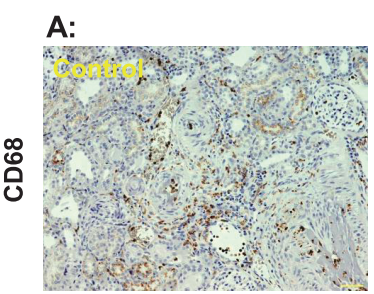

D:

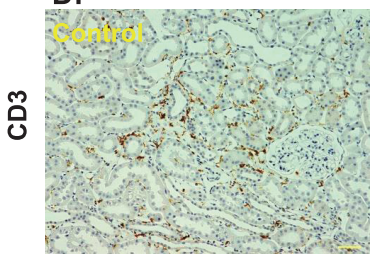

G:

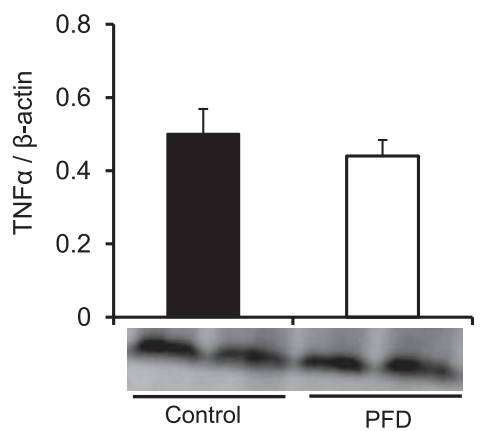

B:

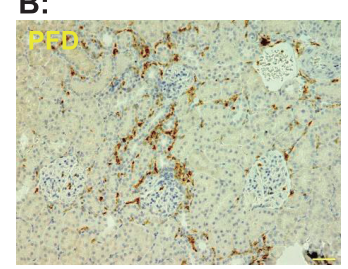

E:

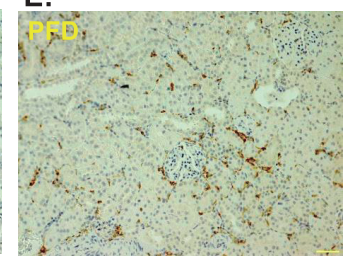

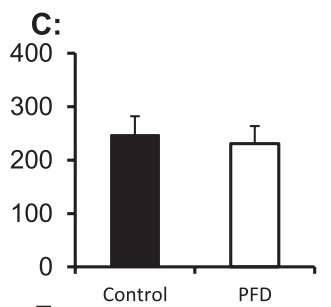

F:

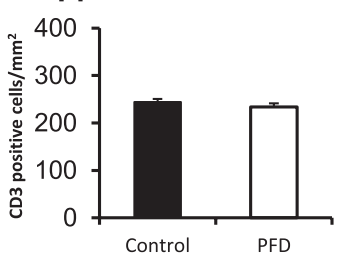

H:

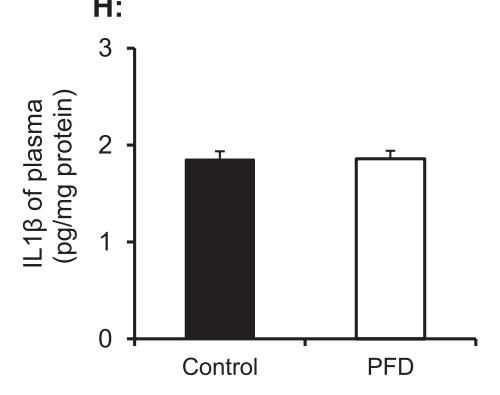

Fig. 6 Effects of PFD treatment on renal inflammatory reaction. Immunohistochemical examination revealed macrophages $(A$ and $B$ ) and $T$ cells ( $D$ and $E$ ), which were identified by positive staining for anti-CD68 and anti-CD3 antibodies, respectively. The numbers of cells positive for CD68 and CD3 were counted and compared between the Control and PFD groups. PFD treatment did not markedly attenuate macrophage (C) or T-cell (F) infiltration. Western blotting analysis also showed that PFD treatment did not significantly attenuate renal TNFa protein expression (G). ELISA revealed that PFD treatment did not affect the plasma level of IL1 $\beta(H)$. ( $n=10$ for Control and PFD treatment groups) 
A:

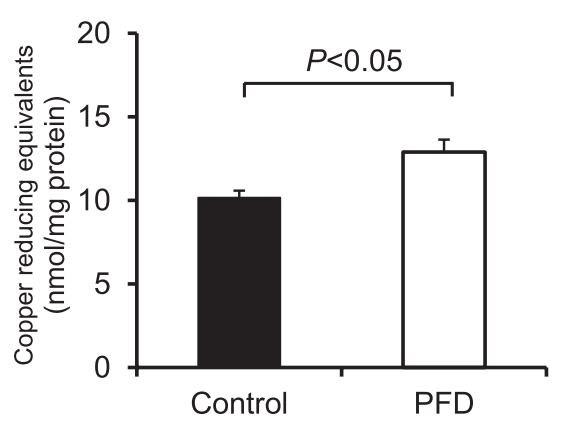

B:

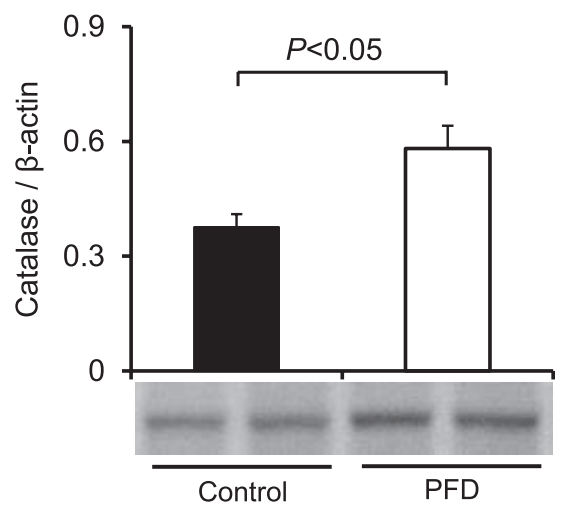

Fig. 7 Effects of PFD treatment on antioxidant activity. Total serum antioxidant activity was measured using the total antioxidant power kit. The activity level (expressed as copper-reducing equivalents) was significantly higher in the PFD group than the Control group after 6 weeks of high-salt diet intake $(A)$. Western blotting showed that catalase expression was significantly higher in the PFD group than the Control group (B). ( $n=10$ for Control and PFD groups)

plays an important role in protecting renal function (21). Moreover, PFD affects MMP9/TIMP1 balance $(6,10,20,36)$. In the present study, PFD treatment increased MMP9 expression and decreased TIMP1 expression, suggesting that it may increase extracellular matrix degradation, leading to the attenuation of renal fibrosis.

PFD is also reported to attenuate renal fibroblast activation and proliferation in vitro (14). In the present study, PFD treatment significantly attenuated the number of FSP1-positive cells compared to the Control group. These results suggest that PFD treatment attenuates fibroblast proliferation; this may represent another possible mechanism of the anti-fibrotic effect of PFD.

From the results described above, we conclude that PFD exerts its anti-fibrotic effect via three mechanisms: 1) downregulation of TGF $\beta$-Smad $2 / 3$ signaling, 2) improvement of MMP9/TIMP1 balance, and 3) suppression of fibroblast proliferation (Fig. 8).

The association between renal inflammation and the development of renal fibrosis has been demonstrated in chronic kidney disease models $(15,16$, 29). Moreover, PFD reduces the production of inflammatory mediators such as $\mathrm{TNF} \alpha$ and $\operatorname{IL} 1 \beta(2$, $12,13,20)$. Therefore, we also investigated the effects of PFD on inflammation in hypertensioninduced renal injury. Unexpectedly, there were no significant differences between the Control and PFD groups with respect to macrophage or T-cell infiltration, namely $\mathrm{TNF} \alpha$ protein expression or plasma levels of IL1 $\beta$. From these results, we conjecture that PFD ameliorates the initial inflammation; how- ever, if the injury continues toward end-stage renal disease, the anti-inflammatory effect of PFD is insufficient. Furthermore, the anti-fibrotic effect of PFD is the main mechanism that improves renal function.

Oxidative stress is also involved in progressive renal injury (28). Catalase deficiency promotes oxidant renal injury and fibrosis in mice (19). Moreover, PFD inhibited the production of reactive oxygen species in mesangial cells (27). We also observed that PFD treatment significantly increased renal catalase expression and total serum antioxidant activity. Therefore, we conclude that the anti-oxidative stress effect of PFD ameliorates renal injury and fibrosis.

Renal fibrosis can be induced via several mechanisms in different renal diseases but it ultimately produces identical fibrotic changes in the kidneys. In hypertension-induced renal injury, the therapeutic mechanisms of PFD are mediated by its anti-fibrotic and anti-oxidative stress pathways, not its anti-inflammatory pathway. PFD exerts its anti-fibrotic effect via three mechanisms: 1) the downregulation of TGF $\beta$-Smad2/3 signaling, 2) improvement of MMP9/TIMP1 balance, and 3) suppression of fibroblast proliferation (Fig. 8). TGF $\beta$ is a central regulator of renal fibrosis and is inhibited by PFD. Furthermore, PFD is a promising agent for the prevention of hypertensive renal injury. The clinical effectiveness of PFD has been evaluated in human diabetic nephropathy (32) and focal segmental glomerulosclerosis (7). Clarifying the renoprotective mechanisms of PFD will help us create new therapeutic strategies and could confer further benefits beyond the therapies currently used for the treat- 


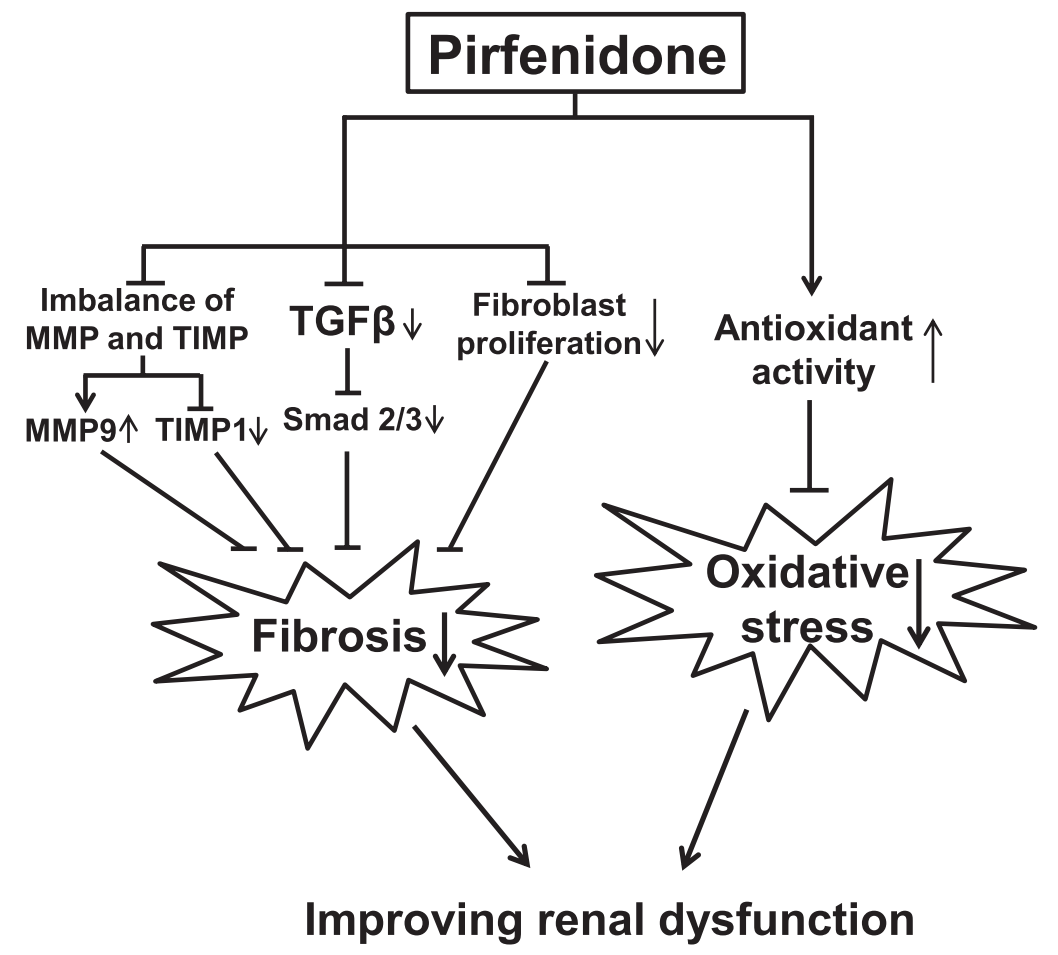

Fig. 8 The renoprotective mechanisms of PFD in hypertensive renal injury: through anti-fibrotic and anti-oxidative stress pathways. The therapeutic mechanisms of PFD on renal injury are mediated by its anti-fibrotic and anti-oxidative stress activities, not its anti-inflammatory activity. PFD exerts its anti-fibrotic effect via three pathways: (1) downregulation of TGF $\beta$ Smad2/3 signaling, (2) improvement of MMP9/TIMP1 balance, and (3) suppression of fibroblast proliferation. TGF $\beta$ is a central regulator of renal fibrosis and is inhibited by PFD.

ment of chronic renal diseases.

\section{Acknowledgements}

We express our gratitude to Dr. Tomoko Ohya and Dr. Kyoko Shioya for their help. This work was supported in part by the Adaptable and Seamless Technology Transfer Program through Target-driven R\&D, Japan (AS232Z02131G); the Program for the Promotion of Fundamental Studies in Health Science of the National Institute of Biomedical Innovation, Japan (Project ID: 10-24); the Grant-in-Aid for Scientific Research (C), Japan (23590705); the Health and Labour Sciences Research Grants, Japan (H24food-004); and the Advanced Research for Medical Products Mining Programme of the National Institute of Biomedical Innovation (NIBIO), Japan.

\section{REFERENCES}

1. Al-Bayati MA, Xie Y, Mohr FC, Margolin SB and Giri SN (2002) Effect of pirfenidone against vanadate-induced kidney fibrosis in rats. Biochem pharm 64, 517-525.

2. Arumugam TV, Shiels IA, Margolin SB, Taylor SM and Brown L (2002) Pirfenidone attenuates ischaemia-reperfusion injury in the rat small intestine. Clin Exp Pharmacol physiol 29, 996-1000.

3. Border WA, Okuda S, Languino LR, Sporn MB and Ruoslahti E (1990) Suppression of experimental glomerulonephritis by antiserum against transforming growth factor beta 1 . Nature 346, 371-374.

4. Bottinger EP and Bitzer M (2002) TGF-beta signaling in renal disease. J Am Soc Nephrol 13, 2600-2610.

5. Brook NR, Waller JR, Bicknell GR and Nicholson ML (2005) The experimental agent pirfenidone reduces pro-fibrotic gene expression in a model of tacrolimus-induced nephrotoxicity. J Surg Res 125, 137-143.

6. Brook NR, Waller JR, Bicknell GR and Nicholson ML (2005) The novel antifibrotic agent pirfenidone attenuates the profibrotic environment generated by calcineurin inhibitors in the rat salt-depletion model. Transplant Proc 37, 130-133.

7. Cho ME, Smith DC, Branton MH, Penzak SR and Kopp JB (2007) Pirfenidone slows renal function decline in patients with focal segmental glomerulosclerosis. Clin J Am Soc Nephrol 2, 906-913.

8. Chromek M, Tullus K, Hertting O, Jaremko G, Khalil A, Li YH and Brauner A (2003) Matrix metalloproteinase-9 and tissue inhibitor of metalloproteinases-1 in acute pyelonephritis and renal scarring. Pediatr Res 53, 698-705.

9. Derynck R and Zhang YE (2003) Smad-dependent and Smad-independent pathways in TGF-beta family signalling. Nature 425, 577-584.

10. Di Sario A, Bendia E, Macarri G, Candelaresi C, Taffetani S, Marzioni M, Omenetti A, De Minicis S, Trozzi L and 
Benedetti A (2004) The anti-fibrotic effect of pirfenidone in rat liver fibrosis is mediated by downregulation of procollagen alpha1(I), TIMP-1 and MMP-2. Dig Liver Dis 36, 744 751.

11. Gonzalez-Avila G, Iturria C, Vadillo-Ortega F, Ovalle C and Montano M (1998) Changes in matrix metalloproteinases during the evolution of interstitial renal fibrosis in a rat experimental model. Pathobiology 66, 196-204.

12. Grattendick KJ, Nakashima JM, Feng L, Giri SN and Margolin SB (2008) Effects of three anti-TNF-alpha drugs: etanercept, infliximab and pirfenidone on release of TNF-alpha in medium and TNF-alpha associated with the cell in vitro. Int immunopharmacol 8, 679-687.

13. Hale ML, Margolin SB, Krakauer T, Roy CJ and Stiles BG (2002) Pirfenidone blocks the in vitro and in vivo effects of staphylococcal enterotoxin B. Infect Immun 70, 2989-2994.

14. Hewitson TD, Kelynack KJ, Tait MG, Martic M, Jones CL, Margolin SB and Becker GJ (2001) Pirfenidone reduces in vitro rat renal fibroblast activation and mitogenesis. $J$ Nephrol 14, 453-460.

15. Ji X, Naito Y, Hirokawa G, Weng H, Hiura Y, Takahashi R and Iwai N (2012) P2X(7) receptor antagonism attenuates the hypertension and renal injury in Dahl salt-sensitive rats. $\mathrm{Hy}$ pertens Res 35, 173-179.

16. Ji X, Naito Y, Weng H, Endo K, Ma X and Iwai N (2012) $\mathrm{P} 2 \mathrm{X} 7$ deficiency attenuates hypertension and renal injury in deoxycorticosterone acetate (DOCA)-salt hypertension. Am J Physiol 308, F1207-2015.

17. Ji X, Takahashi R, Hiura Y, Hirokawa G, Fukushima Y and Iwai N (2009) Plasma miR-208 as a biomarker of myocardial injury. Clin Chem 55, 1944-1949.

18. Jin Y, Ratnam K, Chuang PY, Fan Y, Zhong Y, Dai Y, Mazloom AR, Chen EY, D'Agati V, Xiong H, Ross MJ, Chen N, Ma'ayan A and He JC (2012) A systems approach identifies HIPK2 as a key regulator of kidney fibrosis. Nat Med 18, 580-588.

19. Kobayashi M, Sugiyama H, Wang DH, Toda N, Maeshima Y, Yamasaki Y, Masuoka N, Yamada M, Kira S and Makino H (2005) Catalase deficiency renders remnant kidneys more susceptible to oxidant tissue injury and renal fibrosis in mice. Kidney Int 68, 1018-1031.

20. Lee KW, Everett THt, Rahmutula D, Guerra JM, Wilson E, Ding C and Olgin JE (2006) Pirfenidone prevents the development of a vulnerable substrate for atrial fibrillation in a canine model of heart failure. Circulation 114, 1703-1712.

21. Lelongt B, Bengatta S, Delauche M, Lund LR, Werb Z and Ronco PM (2001) Matrix metalloproteinase 9 protects mice from anti-glomerular basement membrane nephritis through its fibrinolytic activity. $J$ Exp Med 193, 793-802.

22. Ludwig S, Engel K, Hoffmeyer A, Sithanandam G, Neufeld B, Palm D, Gaestel M and Rapp UR (1996) 3pK, a novel mitogen-activated protein (MAP) kinase-activated protein kinase, is targeted by three MAP kinase pathways. Mol Cell Biol 16, 6687-6697.

23. McMillan JI, Riordan JW, Couser WG, Pollock AS and Lovett DH (1996) Characterization of a glomerular epithelial cell metalloproteinase as matrix metalloproteinase-9 with enhanced expression in a model of membranous nephropathy. $J$ Clin Invest 97, 1094-1101.

24. Miric G, Dallemagne C, Endre Z, Margolin S, Taylor SM and Brown L (2001) Reversal of cardiac and renal fibrosis by pirfenidone and spironolactone in streptozotocin-diabetic rats. Br J Pharmacol 133, 687-694.

25. Mo W, Brecklin C, Garber SL, Song RH, Pegoraro AA, Au J, Arruda JA, Dunea G and Singh AK (1999) Changes in collagenases and TGF-beta precede structural alterations in a model of chronic renal fibrosis. Kidney Int 56, 145-153.

26. Piek E, Moustakas A, Kurisaki A, Heldin $\mathrm{CH}$ and ten Dijke P (1999) TGF-(beta) type I receptor/ALK-5 and Smad proteins mediate epithelial to mesenchymal transdifferentiation in NMuMG breast epithelial cells. J Cell Sci 112 (Pt 24), 4557-4568.

27. RamachandraRao SP, Zhu Y, Ravasi T, McGowan TA, Toh I, Dunn SR, Okada S, Shaw MA and Sharma K (2009) Pirfenidone is renoprotective in diabetic kidney disease. J Am Soc Nephrol 20, 1765-1775.

28. Reddi AS and Bollineni JS (2001) Selenium-deficient diet induces renal oxidative stress and injury via TGF-beta1 in normal and diabetic rats. Kidney Int 59, 1342-1353.

29. Rodriguez-Iturbe B, Pons H, Herrera-Acosta J and Johnson RJ (2001) Role of immunocompetent cells in nonimmune renal diseases. Kidney Int 59, 1626-1640.

30. Salazar-Montes A, Ruiz-Corro L, Lopez-Reyes A, CastrejonGomez E and Armendariz-Borunda J (2008) Potent antioxidant role of pirfenidone in experimental cirrhosis. Eur $J$ Pharmacol 595, 69-77.

31. Schiffer M, Schiffer LE, Gupta A, Shaw AS, Roberts IS, Mundel P and Bottinger EP (2002) Inhibitory Smads and TGF-Beta signaling in glomerular cells. J Am Soc Nephrol 13, 2657-2666.

32. Sharma K, Ix JH, Mathew AV, Cho M, Pflueger A, Dunn SR, Francos B, Sharma S, Falkner B, McGowan TA, Donohue M, Ramachandrarao S, Xu R, Fervenza FC and Kopp JB (2011) Pirfenidone for diabetic nephropathy. J Am Soc Nephrol 22, 1144-1151.

33. Sharma K, Ziyadeh FN, Alzahabi B, McGowan TA, Kapoor S, Kurnik BR, Kurnik PB and Weisberg LS (1997) Increased renal production of transforming growth factor-betal in patients with type II diabetes. Diabetes 46, 854-859.

34. Shihab FS, Bennett WM, Yi H and Andoh TF (2002) Pirfenidone treatment decreases transforming growth factor-betal and matrix proteins and ameliorates fibrosis in chronic cyclosporine nephrotoxicity. Am J Transplant 2, 111-119.

35. Shimizu T, Fukagawa M, Kuroda T, Hata S, Iwasaki Y, Nemoto M, Shirai K, Yamauchi S, Margolin SB, Shimizu F and Kurokawa K (1997) Pirfenidone prevents collagen accumulation in the remnant kidney in rats with partial nephrectomy. Kidney Int Suppl 63, S239-243.

36. Shimizu T, Kuroda T, Hata S, Fukagawa M, Margolin SB and Kurokawa K (1998) Pirfenidone improves renal function and fibrosis in the post-obstructed kidney. Kidney Int 54, 99109.

37. Strutz F, Okada H, Lo CW, Danoff T, Carone RL, Tomaszewski JE and Neilson EG (1995) Identification and characterization of a fibroblast marker: FSP1. J Cell Biol 130, 393-405.

38. Takakuta K, Fujimori A, Chikanishi T, Tanokura A, Iwatsuki Y, Yamamoto M, Nakajima H, Okada M and Itoh H (2009) Renoprotective properties of pirfenidone in subtotally nephrectomized rats. Eur J Pharmacol 629, 118-124.

39. Wang S, Wilkes MC, Leof EB and Hirschberg R (2005) Imatinib mesylate blocks a non-Smad TGF-beta pathway and reduces renal fibrogenesis in vivo. FASEB $J 19,1-11$. 